This item is the archived peer-reviewed author-version of:

Layered silicate clays as templates for anisotropic gold nanoparticle growth

\title{
Reference:
}

Hill Eric H., Claes Nathalie, Bals Sara, Liz-Marzan Luis M..- Layered silicate clays as templates for anisotropic gold nanoparticle growth

Chemistry of materials - ISSN 0897-4756 - 28:14(2016), p. 5131-5139

Full text (Publisher's DOI): http://dx.doi.org/doi:10.1021/ACS.CHEMMATER.6B02186

To cite this reference: http://hdl.handle.net/10067/1351780151162165141 
This document is confidential and is proprietary to the American Chemical Society and its authors. Do not copy or disclose without written permission. If you have received this item in error, notify the sender and delete all copies.

\section{Layered Silicate Clays as Templates for Anisotropic Gold Nanoparticle Growth}

\begin{tabular}{|r|l|}
\hline Journal: & Chemistry of Materials \\
\hline Manuscript ID & cm-2016-021868.R1 \\
\hline Manuscript Type: & Article \\
\hline Complete List of Authors: & $\begin{array}{l}\text { Hill, Eric; Centro de Investigacion Cooperativa en Biomateriales } \\
\text { Claes, Nathalie; EMAT, University of Antwerp, } \\
\text { Bals, Sara; EMAT-University of Antwerp, Physics } \\
\text { Liz-Marzán, Luis; CIC biomaGUNE, ; }\end{array}$ \\
\hline & \\
\hline
\end{tabular}

\section{SCHOLARONE ${ }^{\text {m }}$ \\ Manuscripts}




\title{
Layered Silicate Clays as Templates for Anisotropic Gold Nanoparticle Growth
}

\author{
Eric H. Hill ${ }^{1}$, Nathalie Claes ${ }^{2}$, Sara Bals ${ }^{2}$, Luis M. Liz-Marzán ${ }^{1,3}$ \\ ${ }^{1}$ Bionanoplasmonics Laboratory, CIC biomaGUNE, 20009 Donostia-San Sebastián, \\ Spain \\ ${ }^{2}$ EMAT-University of Antwerp, Groenenborgerlaan 171, B-2020 Antwerp, Belgium \\ ${ }^{3}$ Ikerbasque, Basque Foundation for Science, 48013 Bilbao, Spain
}

\begin{abstract}
Clay minerals are abundant natural materials arising in the presence of water, and are composed of small particles of different sizes and shapes. The interlamellar space between layered silicate clays can also be used to host a variety of different organic and inorganic guest molecules or particles. Recent studies of clay-metal hybrids formed by impregnation of nanoparticles into the interlayer spaces of the clays have not demonstrated the ability for templated growth following the shape of the particles. Following this line of interest, a method for the synthesis of gold nanoparticles on the synthetic layered silicate clay laponite was developed. This approach can be used to make metal-clay nanoparticles with a variety of morphologies while retaining the molecular adsorption properties of the clay. The SERS enhancement of these particles was also found to be greater than that obtained from metal nanoparticles of a similar morphology, likely due to increased dye adsorption from the presence of the clay. The hybrid particles presented herein will contribute to further study of plasmonic sensing, catalysis, dye aggregation, and novel composite materials.
\end{abstract}




\section{Introduction}

Metal nanoparticles (NPs) are of great interest due to their myriad applications arising from nanoscale properties, which deviate from those of bulk materials. ${ }^{1}$ Noble metals are particularly interesting and have been well-studied due to their shape- and size-dependent physical and electronic properties. ${ }^{2}$ Many of these properties are granted or influenced by the occurrence of plasmons, which are oscillations of the electron density in the nanoparticle upon excitation by light. ${ }^{3}$ In addition to non-linear optical effects and improved catalysis, surface plasmons can greatly enhance the scattering of molecules in the vicinity of the metal surface where the plasmon is generated, and this effect has given rise to highly-sensitive analytical techniques such as surface enhanced Raman scattering (SERS), which can even reach single-molecule detection under certain conditions. ${ }^{4}$ SERS alone has led to a plethora of interesting advances in sensing of molecular species, ions, ${ }^{5}$ disease biomarkers, and other compounds, leading to breakthroughs in medical diagnostics, ${ }^{6}$ monitoring of environmental pollutants, ${ }^{7}$ and monitoring the dynamics of bacterial communication. ${ }^{8}$ In addition to analytical techniques, metal nanoparticles have been shown to have improved catalytic properties compared with bulk. ${ }^{9}$ A number of reviews cover the history of these developments, which have guided the synthetic efforts of metal NPs over the last 20 years., ${ }^{1,9-11}$ Through control of the twinning and crystal structure of metal seed particles, the growth of the particle along different crystal facets can be directed to form anisotropic shapes such as triangles and rods. ${ }^{12,13}$ Recently, studies on the use of template materials for the growth of metal nanoparticles to conform to a certain shape have been performed using inorganic templates such as silica and biological templates such as DNA and viral capsids, though many of these techniques still rely on the use of seeds for some part of the synthesis. ${ }^{9,10}$ Clay particles have also been used to direct nanoparticle growth, ${ }^{14}$ with recent emphasis on layered silicate clays due to their interesting electrostatic properties and anisotropic shapes.

Clay minerals are abundant natural materials arising in the presence of water, which can be dispersed in liquids to form suspensions consisting of particles with a variety of sizes and shapes such as rods ${ }^{15}$ and plates. ${ }^{16}$ These clay particles are able to self-organize into complex structure ${ }^{17}$ and can have rheological effects resulting in a variety of phases such as gels ${ }^{18}$, glasses, ${ }^{19,20}$ and "ringing" gels. ${ }^{21}$ In addition to their abundance and low cost, properties of clays such as high surface area, good ion- 
exchange capacity, and recyclability have led to their use in numerous industrial processes. Layered silicate clays, such as montmorillonite and laponite, can delaminate in water to a suspension of platelets with a high aspect ratio (300:1). ${ }^{19,22-24}$ Studies have shown that through loading of organic molecules on clay particles, the intermolecular interactions and resulting molecular aggregates can be altered. ${ }^{25}$ This can result in different photophysical and chemical properties leading to alteration of photochemical processes $^{26,27}$ such as the enhancement of organic laser dyes ${ }^{25}$ and reducing photochemical degradation. ${ }^{28}$ Additionally, their potential biomedical applications, such as drug-delivery and release and wound healing, have been the subject of recent study. ${ }^{29-33}$ We expect that the combination of plasmonic properties of anisotropic metal nanoparticles with the molecular adsorption of the clay will lead to improved SERS enhancement over a purely metal particle.

Laponite RD (laponite) is a synthetic layered silicate clay with the chemical formula $\mathrm{Na}^{+0.7}\left[\left(\mathrm{Si}_{8} \mathrm{Mg}_{5.5} \mathrm{Li}_{0.3}\right) \mathrm{O}_{20}(\mathrm{OH})_{4}\right]^{-0.7}$. Its specific surface area determined by nitrogen adsorption is $370 \mathrm{~m}^{2} / \mathrm{g}$, and its density is $\sim 2.53 \mathrm{~g} / \mathrm{cm}^{3} .{ }^{34}$ A smectite-type clay, it is composed of disc-like particles with a thickness of $\sim 1 \mathrm{~nm}$ and a diameter of $25-30 \mathrm{~nm}^{35}$ While the cation exchange capacity is $0.75 \mathrm{meq} / \mathrm{g}$, the discs can be charged heterogeneously in aqueous suspensions depending on the $\mathrm{pH} .{ }^{36}$ While similar to other natural smectite clays such as montmorillonite, the synthetic nature of laponite provides a clay with a narrower distribution of particle diameter and cation exchange capacity. Scheme 1 gives a visual representation of laponite and its crystal structure.
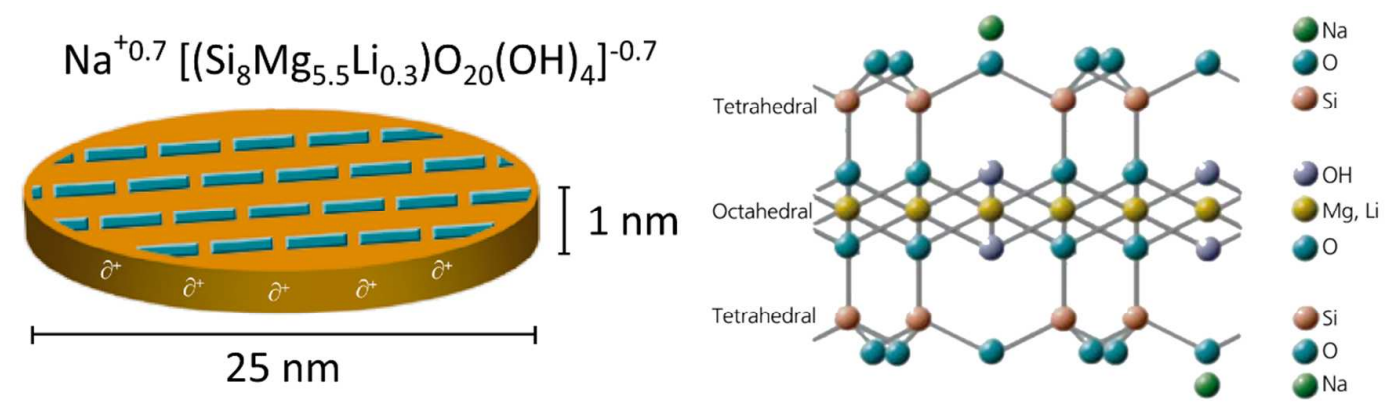

Scheme 1. Schematic view of the morphology, surface charge distribution and crystal structure of a laponite clay particle. 
While previous studies have demonstrated the potential for layered silicate clays to be used as supports for metal nanoparticle growth and intercalation, ${ }^{26,27}$ the ability to use the delaminated clay particles as shaped templates for nanoparticle growth has not been achieved. In previous studies, the resulting particles are either spherical and/or small $(<10 \mathrm{~nm})$, due to particle growth in the interlamellar spaces of the clay. ${ }^{26,27}$ In this case, the growth of the metal crystal is not influenced by the discoidal shape of the clay, which we found of interest toward the preparation of composite metal-clay particles, in particular when using fully delaminated discoidal clay particles. Following this line of interest, the synthesis of gold nanoparticles on the synthetic layered silicate clay laponite was developed in this work.

\section{Materials and Methods}

\section{Materials}

Cetyltrimethylammonium chloride $25 \%$ (w/w) (CTAC), $\mathrm{HAuCl}_{4}(99.99 \%$ ), L-ascorbic acid (99\%) (AA), sodium hydroxide (98\%), and hydrochloric acid (37\%) were obtained from Sigma-Aldrich and used without further purification. Laponite RD was graciously provided by BYK additives. All solutions and dispersions were prepared using Millipore-filtered water with a resistivity of $18.2 \mathrm{M} \Omega \cdot \mathrm{cm}$. Dispersions of laponite were prepared at $1 \mathrm{mg} / \mathrm{mL}$, sonicated in a bath-type sonicator (200W, Ultrasons-H, JP SELECTA) for an hour, and allowed to sit for 24 hours prior to use.

\section{Synthesis of Au-Laponite nanoparticles}

In a typical synthesis, the following procedure was followed, but synthesis parameters were systematically varied, such as reagent concentrations, temperature $(0,28$, and 60 $\left.{ }^{\circ} \mathrm{C}\right)$, and $\mathrm{pH}$. Laponite dispersion $(8 \mathrm{~mL}, 1 \mathrm{mg} / \mathrm{mL}$ ) was diluted to $100 \mathrm{~mL}$ with water, and $\mathrm{HAuCl}_{4}(10 \mu \mathrm{L}, 126 \mathrm{mM})$ was then added under constant stirring, followed by 100 $\mu \mathrm{L}$ of $25 \%$ CTAC $(754 \mathrm{mM})$ and the solution was sonicated for 5 minutes. Upon removal from the sonication bath, the solution was returned to the stirring plate and AA $(100 \mu \mathrm{L}, 127 \mathrm{mM})$ was added under vigorous stirring. The $\mathrm{pH}$ of the laponite dispersion was typically 7.4, however to explore the effects of $\mathrm{pH}$ on charging of the edges of the laponite discs, reactions were also carried out with solution $\mathrm{pH}$ adjusted to 5.1, 3.1, 2.8, 
and 2.5, using $0.1 \mathrm{M} \mathrm{HCl}$. An additional synthesis was carried out to study the influence of a long stirring time (rather than sonication of laponite with gold). To this end, laponite dispersions of $1 \mathrm{mg} / \mathrm{mL}, 0.66 \mathrm{mg} / \mathrm{mL}, 0.33 \mathrm{mg} / \mathrm{mL}$, and $0.1 \mathrm{mg} / \mathrm{mL}$ were stirred overnight with a magnetic stirrer at $700 \mathrm{rpm}$ and $25^{\circ} \mathrm{C}$. Rapid stirring was applied prior to adding ascorbic acid. The appearance of a blue color within seconds of AA addition indicated particle formation, and the synthesis was deemed complete within minutes of the color formation. Particles were rinsed by centrifugation at 6500 rpm followed by redispersion in water.

\section{Characterization}

UV-Visible absorbance spectra of particles dispersed in water were recorded with an Agilent 8543 UV-visible spectrophotometer. Dynamic light scattering and zeta-potential were measured using a Malvern Instruments Zetasizer Nano S. Samples for electron microscopy were prepared by vacuum-drying of a small volume (1-10 $\mu \mathrm{L})$ of a particle dispersion onto the carbon film of a copper Transmission Electron Microscopy (TEM) grid (400 mesh size carbon film). Initial TEM characterization was performed using a JEOL JEM-1400PLUS with an accelerating voltage of $120 \mathrm{kV}$. Advanced electron microscopy, including Energy Dispersive X-ray Spectroscopy (EDX), was performed using a FEI Tecnai operated at $200 \mathrm{kV}$. Surface enhanced Raman scattering (SERS) measurements were performed on a confocal Raman microscope (micro-Renishaw InVia Reflex system equipped with Peltier charge-coupled device (CCD) detectors) using a $10 \times$ objective (N.A. $=0.85)$ with an excitation wavelength of either $633 \mathrm{~nm}$ with 1800 lines/mm diffraction grating, or $785 \mathrm{~nm}$ with a 1200 lines/mm grating. $\mathrm{P} \approx$ $0.15 \mathrm{~mW}$, integration time $10 \mathrm{~s}$. Samples were prepared in glass vials with a total volume of $1 \mathrm{~mL}$ of Millipore-filtered water and $1 \mu \mathrm{M}$ of either 4-mercaptobenzoic acid, 1-Napthalene thiol, 2-napthalene thiol, or crystal violet. [ $\left.\mathrm{Au}^{0}\right]$ was $0.25 \mathrm{mM}$ for all particles tested. SERS results for laponite-gold particles were compared with surfactantfree gold nanostars synthesized by a published seed-mediated growth method. ${ }^{37}$

\section{Results and Discussion}

Surfactant-free AuNPs on Laponite Clay 
The formation of gold nanoparticles on the layered silicate clay, Laponite RD, was performed by diluting laponite in water, sonicating it together with $\mathrm{HAuCl}_{4}$, and adding ascorbic acid under stirring. In order to examine the effects of sonication, nanoparticles were also synthesized with the sonication step replaced by overnight stirring. Experimental details are described in the Methods section. The strong interaction between $\mathrm{Au}$ and laponite is clear even prior to the reduction step. The absorbance band of $\mathrm{HAuCl}_{4}$ at $310 \mathrm{~nm}$ shifts to $290 \mathrm{~nm}$ in the presence of laponite, and increasing laponite concentration to $1 \mathrm{mg} / \mathrm{mL}$ can even result in complete damping of the band (Figure S1, Supporting Information). This demonstrates formation of an $\mathrm{AuCl}_{3}$ complex with laponite, in analogy with citrate reduction, where substitution of a $\mathrm{Cl}^{-}$ ligand by $\mathrm{OH}$ - groups has been shown to be the initial step. ${ }^{38}$ Following the formation of an $\mathrm{AuCl}_{2} \mathrm{OH}^{-}$complex at the laponite interface, progression towards reduction into an $\mathrm{Au}^{1}$ complex is likely to occur with the aid of $\mathrm{SiO}^{-}$and $\mathrm{SiOH}$ groups on the faces of laponite. The measured laponite size of $\sim 25 \mathrm{~nm}$ with a discoidal shape, and $\zeta$-potential of $-39 \mathrm{mV}$, are consistent with reported parameters (Table $\mathbf{S 1}$ of Supporting Information). ${ }^{31}$ UV-Vis spectra (Figure 1) clearly show that control syntheses using CTAC but not laponite result in formation of spherical AuNPs, as indicated by the narrow absorbance band at $\sim 520 \mathrm{~nm}$. The redshifted absorbance when laponite is involved in the synthesis is related to increased anisotropy of the particle shape. Likewise, the effect of $\left[\mathrm{HAuCl}_{4}\right]$ or amount of dispersed clay on the morphology of the resulting AuNPs can be estimated from the UV-Vis spectra in Figures 1B and 1C, below. 

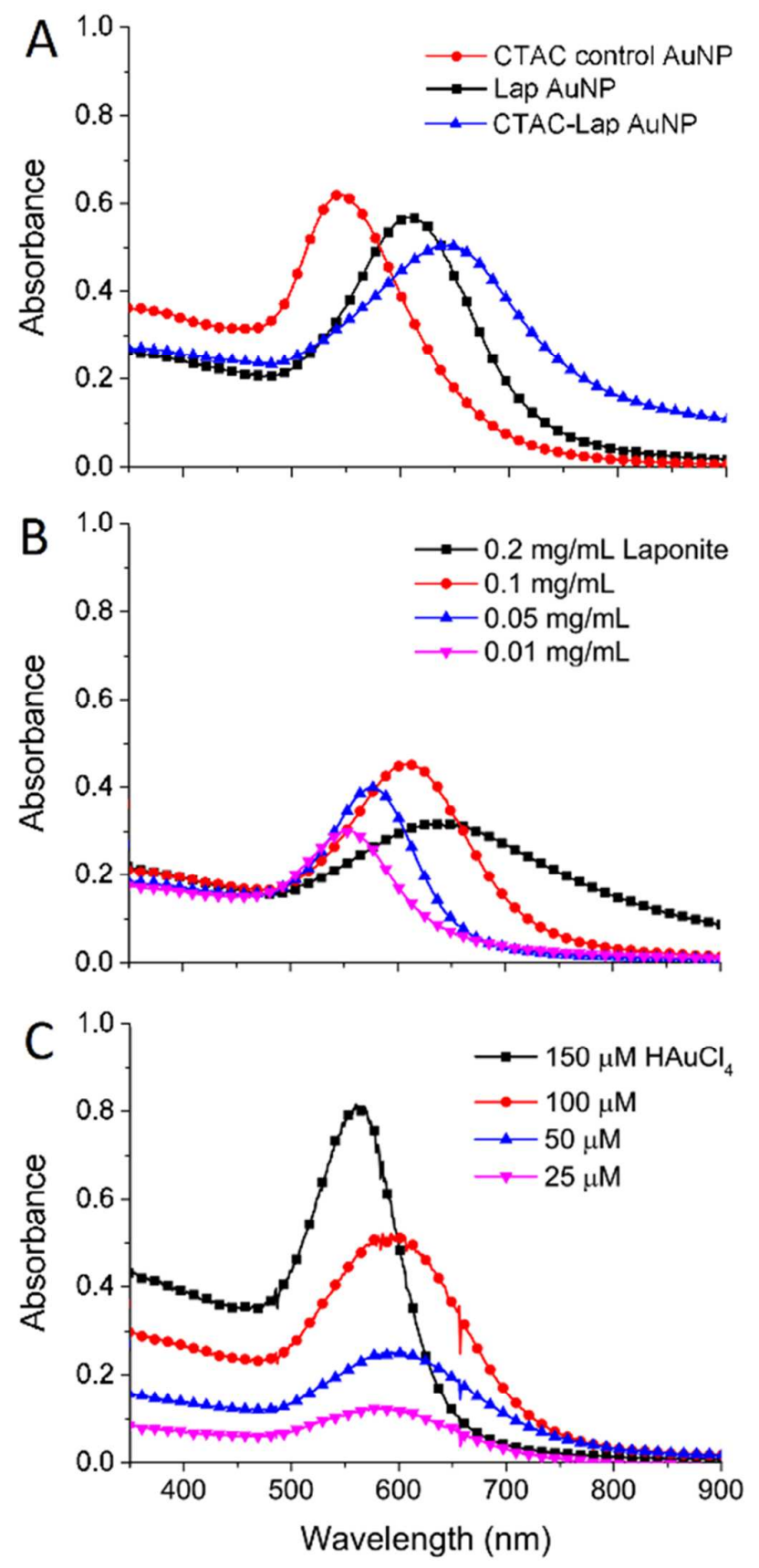

Figure 1. UV-Vis-NIR absorbance spectra of AuNPs grown on laponite clay. (A) CTAC control and particles formed with $0.1 \mathrm{mg} / \mathrm{mL}$ laponite (with and without CTAC); (B) Variation of laponite concentration from 0.01 to $0.2 \mathrm{mg} / \mathrm{mL}$; (C) Variation of $\mathrm{HAuCl}_{4}$ amount with $0.1 \mathrm{mg} / \mathrm{mL}$ laponite.

$\mathrm{Au}^{3+}$ ions in $\mathrm{HAuCl}_{4}$ are likely to associate strongly with the negatively-charged faces of the clay, providing a shell of adsorbed ions which could then be reduced to $\mathrm{Au}^{0}$ at the clay surface upon addition of the reducing agent. One would expect that a high 
gold/clay ratio would result in AuNPs which are more spherical due to the overgrowth of gold beyond a thin layer around the discoidal clay particles or clusters thereof. This is indeed observed in the UV-Vis spectra, where decreased laponite and constant $\mathrm{Au}$ (Fig. 1B) or increased Au with constant laponite (Fig. 1C), both show this trend, i.e. a lowerenergy band and blue color for low gold:laponite ratio and a higher energy absorbance band and more red color for a high gold:laponite ratio. This red-shift of the plasmon band and tailing into the near infrared is clear evidence of the increased anisotropy of the particles, and can be observed for most of the anisotropic particles presented herein.

\section{Influence of surfactant and reducing agent concentration on appendage formation}

The synthesis of anisotropic gold nanoparticles is generally performed with a stabilizing surfactant or polymer, which plays several important roles such as stabilizing specific crystal facets and arresting kinetic growth. ${ }^{11}$ In the case of layered silicates such as laponite, their highly anionic silicate faces have a strong electrostatic interaction with $\mathrm{Au}^{3+}$ and, when CTAC is used as a surfactant, one can also expect strong interaction between the faces of the laponite and the ammonium ions in $\mathrm{CTA}^{+}$. $\mathrm{CTA}^{+}$has been previously used to organically modify layered silicate clays, facilitating delamination of the individual discoidal particles. ${ }^{39,40}$ Therefore, the influence of CTAC on the synthesis of the particles was explored. Interestingly, while previous studies involving CTAB showed flocculation or increased aggregation of laponite particles with CTAB, the addition of CTAC did not result in such flocculation, but instead facilitated delamination at the same ratio of surfactant:laponite. ${ }^{40}$ This suggests that the counterion of $\mathrm{CTA}^{+}$plays a strong role in the delamination of the clay particles, which may be of interest for future studies. In a previous study of the seedless formation of singlecrystal $\mathrm{Au}$ nanostars using CTAC, it was shown that both the CTAC:Au ratio and temperature could be used to control the degree of anisotropy in the particles. ${ }^{41}$ With a high CTAC:Au ratio (120:1), it was found that numerous single-crystal appendages would form. Using the same surfactant, we explored the ability to control the degree to which the clay-AuNPs form anisotropic appendages through variation of CTAC and ascorbic acid concentration. The change in nanoparticles shape was monitored by TEM, as shown in Figure 2. 


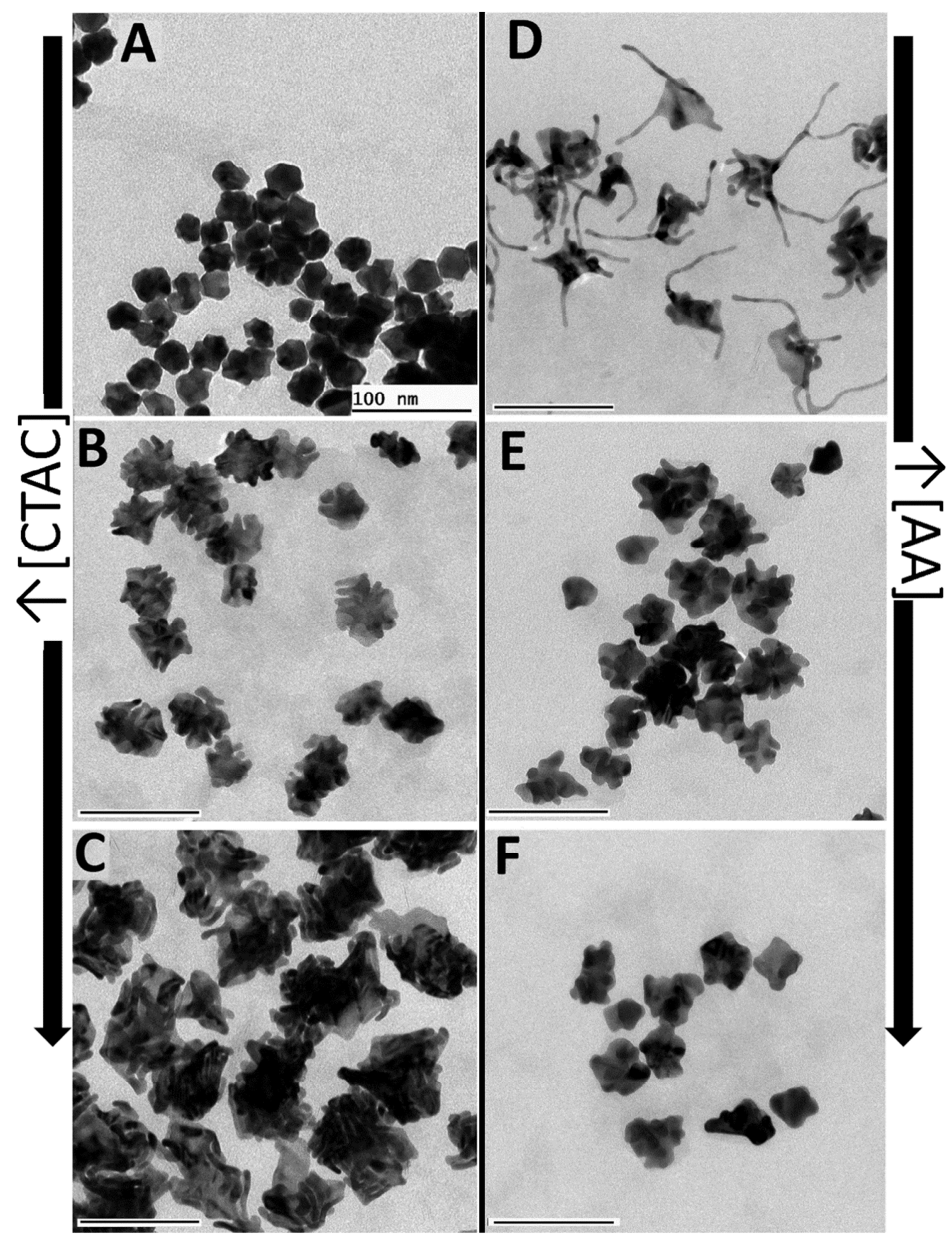

Figure 2. Laponite-Au nanoparticles formed with increasing concentrations of CTAC (A-C) and ascorbic acid (D-F), at constant $[\mathrm{lap}]=0.1 \mathrm{mg} / \mathrm{mL},[\mathrm{Au}]=12.7 \mu \mathrm{M}$. CTAC was varied: $[\mathrm{CTAC}]=0(\mathrm{~A}), 0.37 \mathrm{mM}(\mathrm{B})$, and $1.5 \mathrm{mM}(\mathrm{C})$, keeping $[\mathrm{AA}]=63 \mu \mathrm{M}$. AA was varied: $[\mathrm{AA}]=25 \mu \mathrm{M}(\mathrm{D}), 63 \mu \mathrm{M}(\mathrm{E}), 0.5 \mathrm{mM}(\mathrm{F})$, keeping [CTAC] $=0.37 \mathrm{mM}$. Scale bars are all $100 \mathrm{~nm}$. 
The TEM images in Figure 2 clearly show two significant structural changes upon variations in the amount of CTAC or ascorbic acid added. First, the presence of CTAC results in slightly larger particles. From top to bottom on the left-hand side of Figure 2, it is apparent that with an increase in CTAC the number of finger-like extrusions increases, though they are not extending away from the surface, leading to increased particle size. This is also shown as an increase in absorbance of the plasmon band in the NIR (Figure S2; Supporting information). The influence of reducing agent (AA) concentration on the length of the extrusions was also established. With an AA:Au ratio of 2:1, extrusion length was up to $100 \mathrm{~nm}$ (Figure 2D). As the concentration of AA was increased, the length of the extrusions was reduced, but the total number of extrusions increased. The greatest AA concentration, with an AA:Au ratio of 40:1, resulted in particles with few short extrusions (Figure 2F). The extrusions, particularly in the spider-like particles in Figure 2D, appear to have a central origin which supports the hypothesis that single laponite particles serve as the initial site for metal salt reduction. While these experiments were performed with similar CTAC:AA:Au ratios as a previous study of seedless CTAC particles ${ }^{41}$, the particle morphologies obtained herein are different, and of a more uniform shape and size due to the particle growth being initially confined to the clay template.

Measurement of the $\zeta$-potential allowed us to determine the surface charge of the particles, and thereby estimating the stability of the particles in solution. Bare laponite has a $\zeta$-potential of $-39 \mathrm{mV}$, owing to the strong anionic character of the faces of the disc-like particles. Upon formation of AuNPs with laponite, the $\zeta$-potential increased to $-27 \mathrm{mV}$, as the gold metal coats the anionic faces of the clay disc. For those particles formed with laponite through overnight stirring, the particles were not only found to be larger by dynamic light scattering and TEM (Table S1, Figure S3), but also less stable in solution, with a $\zeta$-potential of only $-18 \mathrm{mV}$. The most stable particles formed on laponite were those where CTAC was involved in the synthesis, with a $\zeta$-potential around $+40 \mathrm{mV}$, the positive charge occurring from the cationic $\mathrm{CTA}^{+}$surrounding the particles. Tabulated values for $\zeta$-potential and particle size by dynamic light scattering are provided in Table S1 of the supporting information. In order to better understand the three-dimensional (3D) morphology of the particles, further characterization was carried out using high angle annular dark field scanning transmission (HAADF-STEM) 
electron tomography. 3D reconstructions of representative particles for the different synthetic approaches are shown in Figure 3.

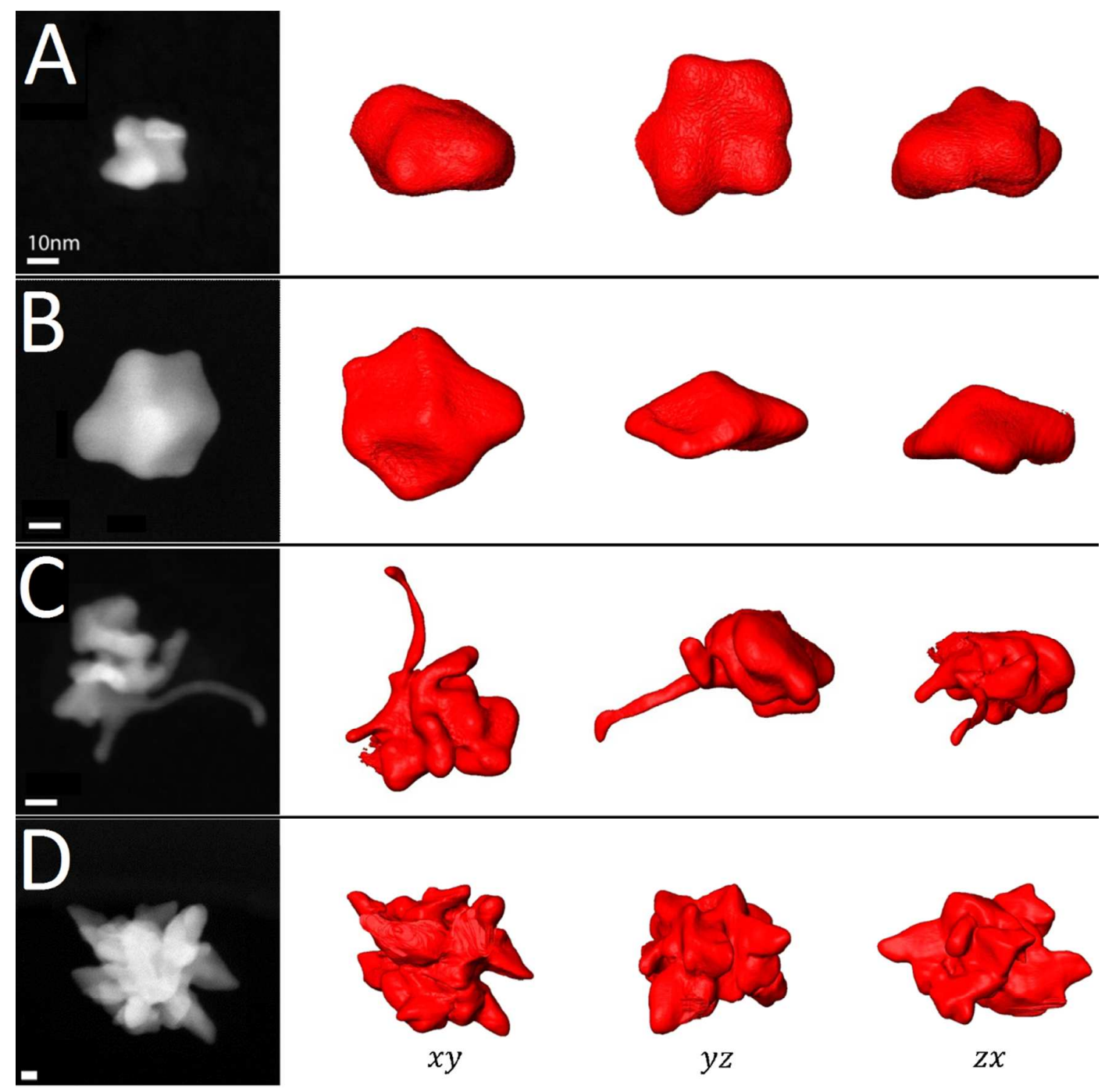

Figure 3. HAADF-STEM and 3D reconstructions for laponite-Au nanoparticles of (A) [lap $]=80 \mathrm{ug} / \mathrm{mL},[\mathrm{Au}]=12.7 \mu \mathrm{M},[\mathrm{AA}]=127 \mathrm{uM}$, similar to Figure 2A; (B) $[\mathrm{lap}]=80$ $\mathrm{ug} / \mathrm{mL},[\mathrm{Au}]=12.7 \mu \mathrm{M},[\mathrm{AA}]=127 \mathrm{uM},[\mathrm{CTAC}]=0.37 \mathrm{mM}$; (C) $[\mathrm{lap}]=80 \mathrm{ug} / \mathrm{mL}$, $[\mathrm{Au}]=12.7 \mu \mathrm{M},[\mathrm{AA}]=25 \mathrm{uM},[\mathrm{CTAC}]=0.37 \mathrm{mM}$, similar to Figure 2D; (D) $[\mathrm{lap}]=1$ $\mathrm{mg} / \mathrm{mL},[\mathrm{Au}]=63.5 \mu \mathrm{M},[\mathrm{AA}]=423 \mu \mathrm{M}$, with $24 \mathrm{~h}$ stirring prior to AA addition. Scale bars are $10 \mathrm{~nm}$.

Electron tomography enables one to observe the particles from different angles, evidencing a flattened aspect for the simpler particles in Figure 3A and 3B. This is 
expected to result from the templating effect of individual silicate particles prior to reduction of adsorbed $\mathrm{Au}$ ions into $\mathrm{Au}^{0}$ by ascorbic acid. In Figure 3C (same particles as in Fig 2D), the appendages coming out of the particle can be clearly observed, in addition to a convoluted central structure. Finally, in Figure 3D the particles synthesized by stirring a relatively high laponite concentration $(1 \mathrm{mg} / \mathrm{mL})$ overnight with gold salt, prior to ascorbic acid addition, are shown to have a richer structure which lends to a high surface area. These particles are also fairly large (80-100 nm), and their complex shape resembles a cluster of particles such as those given in Figures 2A or 3A. This appearance suggests that the stirring time with $\mathrm{Au}^{3+}$ allows the aggregation of multiple disc-like laponite particles into a typical house-of-cards type aggregate prior to reduction. ${ }^{42}$ Additional characterization by TEM and UV-Vis of these particles supports this hypothesis, as the increase in Au:lap ratio in this particular synthesis leads to larger final particles, where the $\mathrm{Au}$ ions may behave as a sort of "glue" to induce laponite aggregation throughout the stirring process (Figure S3). The evolution of particle size with different stirring times was not evaluated, but is expected to follow the previously reported trends in clay aggregate size over time. ${ }^{43}$ An important piece of information regarding the role of the clay discs is provided by EDX measurements of the particles, which reveal the colocalization of $\mathrm{Si}$ and $\mathrm{Mg}$ with $\mathrm{Au}$, thus suggesting the presence of laponite in the particles. A representative example is shown in Figure 4. 


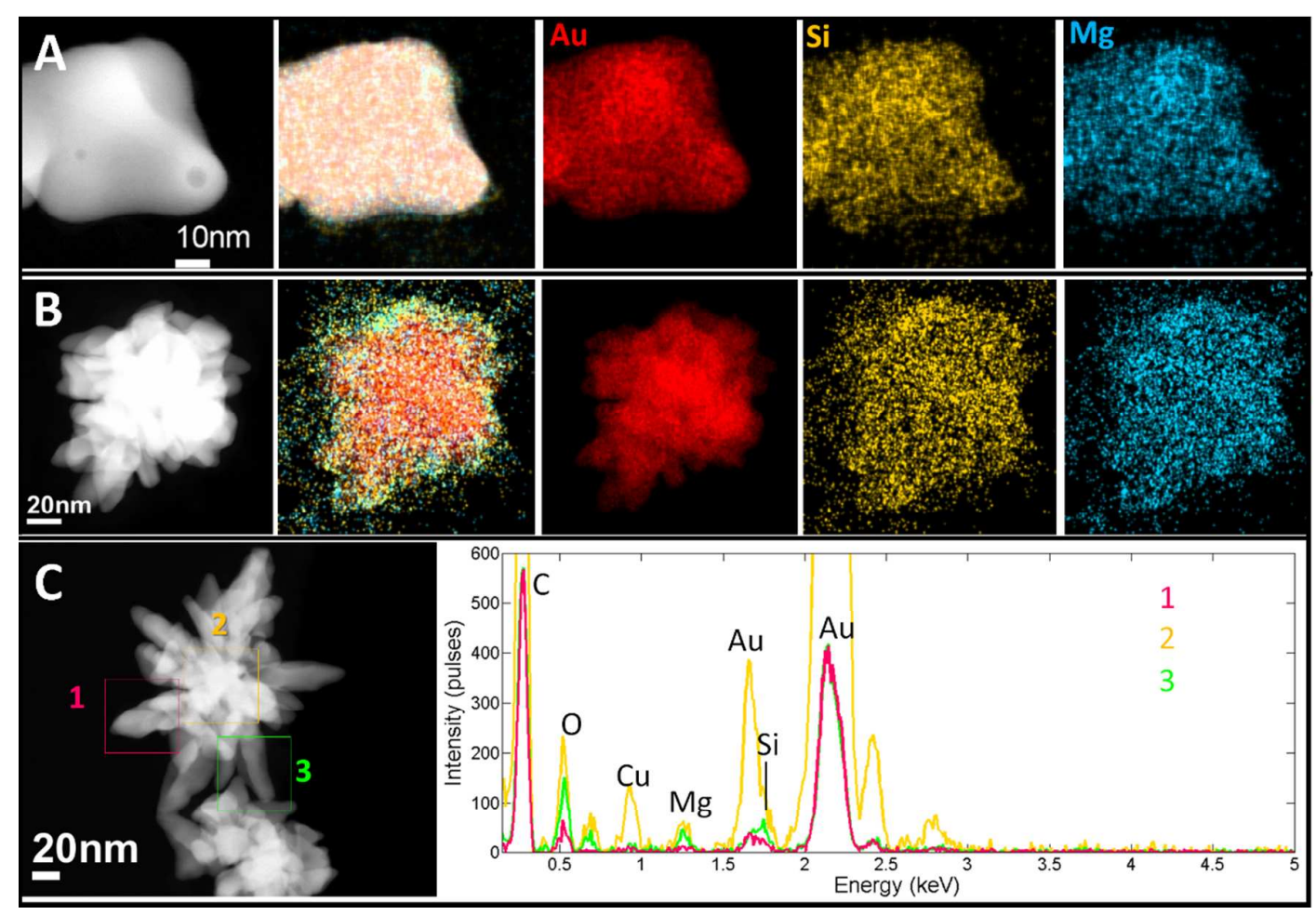

Figure 4. EDX Analysis of lap-Au particles. (A) With CTAC; (B,C) Surfactant-free particles obtained after overnight stirring prior to reduction. $\mathrm{Au}, \mathrm{Mg}$, and $\mathrm{Si}$ are imaged in $\mathrm{A}$ and $\mathrm{B}$. $\mathrm{C}$ shows the difference in elemental composition in different regions of a single particle.

In Figure 4 the EDX analysis of two different types of particles is shown. Both the particles stabilized by CTAC (Figure 3B) and those which were stirred overnight prior to reduction (Figure 3D) showed co-localization of $\mathrm{Au}, \mathrm{Mg}$, and $\mathrm{Si}$. In the case of the overnight-stirred particles, signals from $\mathrm{Mg}$ and $\mathrm{Si}$ are also outside of the $\mathrm{Au}$ region (Figure 4B). This is likely the result of either ablation of the clay causing the signal to spread out, or the aggregation of laponite at the surface of the gold particle. The signal to noise ratios of the $\mathrm{Mg}$ and $\mathrm{Si}$ measurements are much lower than that of $\mathrm{Au}$ due to lower atomic number and some overlap in the EDX peaks of Si and Au. Despite these complications and the low thickness of the clay particles, the $\mathrm{Mg}$ and $\mathrm{Si}$ are co-localized at the expected stoichiometry ( $\mathrm{Si}$ to $\mathrm{Mg}$ 8:5.5).

The influence of $\mathrm{pH}$ on the charge of the edges of laponite discs is well-known, with a $\mathrm{pH}<11$ leading to positively charged edges. ${ }^{43}$ The transition from a neutral to a strongly acidic solution would in turn enhance the edge-face interactions through increased Coloumb interactions, resulting in increased likelihood of the formation of the 
typical "house of cards" type of stacking observed with layered silicate clays. In order to study this effect, the synthesis was carried out with different amounts of $\mathrm{HCl}$ or $\mathrm{NaOH}$ added to the solution prior to sonication with surfactant. In the case of added $\mathrm{NaOH}$, increased rate of particle growth is observed as is commonly known to occur in AuNP syntheses. ${ }^{44}$ On the other hand, the addition of acid to the solution prior to gold reduction strongly influences the particle morphology. In addition to a reduction in the rate of particle growth, also commonly known to occur for AuNP synthesis, increased $\mathrm{HCl}$ concentration led to the formation of particles with highly-branched structure (Figure S4, supporting information). The UV-Vis spectra (Figure S4A) show that as $\mathrm{pH}$ is varied between 3.1 and 2.5 , the plasmon band at $\sim 610 \mathrm{~nm}$ dampens and broadens, whereas the absorption in the infrared increases. The strong IR absorbance shows an increased aspect ratio (higher anisotropy) for the resulting particles. Indeed, when analyzing the TEM images (Figure S4B-D), the formation of highly-branched particles is observed, with the size of a "single particle" dramatically increasing due to the extended branching. Furthermore, the low contrast of the particles apparent in the TEM confirms that the resulting structures are thin enough to allow transmission of some electrons. The low contrast in TEM combined with the strong IR absorbance of these particles shows that they attain a thin, branched particle morphology with only minor changes in acid concentration.

\section{Enhanced SERS Signal of Laponite-Au Nanoparticles}

One of the major uses for gold nanoparticles is in plasmonics, and specifically the utilization of the electric field enhancement for molecular sensing by SERS. We compared the SERS enhancement by laponite-Au nanoparticles to that by well-known surfactant-free nanostars synthesized by a seed-mediated approach. ${ }^{37}$ TEM images and UV-Vis spectra of these particles are shown in Figure $\mathbf{5 5}$ of the Supporting Information. A comparison between surfactant-free laponite-Au nanoparticles and surfactant-free $\mathrm{Au}$ nanostars for detection of $1 \mu \mathrm{M}$ 4-mercaptobenzoic acid (MBA), 1napthalene thiol (1-NaT), and 2-napthalene thiol (2-NaT) is summarized in Figure 5. In each case, the laponite-Au nanoparticles gave increased SERS scattering intensity as compared to $\mathrm{Au}$ nanostars. The prominent peaks of 1-NaT correspond to symmetric $\mathrm{C}$ $\mathrm{H}$ bending $\left(1074 \mathrm{~cm}^{-1}\right)$ and ring stretching $\left(1475 \mathrm{~cm}^{-1}\right)$, with similar modes for 2-NaT at 
$1174 \mathrm{~cm}^{-1}$ and $1481 \mathrm{~cm}^{-1},{ }^{45}$ respectively, and $v(\mathrm{C}-\mathrm{C})\left(1080 \mathrm{~cm}^{-1}\right)$ and ring breathing $\left(1590 \mathrm{~cm}^{-1}\right)$ for MBA. ${ }^{46}$
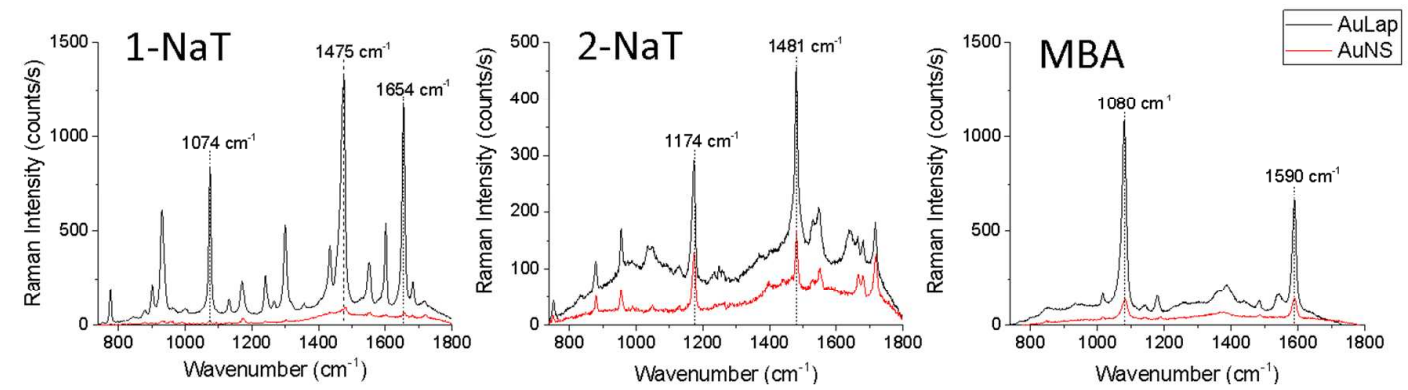

Figure 5. SERS spectra of $1 \mu \mathrm{M} 1-\mathrm{NaT}, 2-\mathrm{NaT}$, and 4-MBA. The black line denotes CTAC-free laponite-gold nanoparticles, while the red line denotes surfactant-free $\mathrm{Au}$ nanostars. The prominent peaks for each Raman dye are noted. [ $\mathrm{Au}^{0}$ ] was $0.25 \mathrm{mM}$ in all cases, particles were synthesized with $[\mathrm{Lap}]=0.1 \mathrm{mg} / \mathrm{mL} ;\left[\mathrm{HAuCl}_{4}\right]=25.4 \mu \mathrm{M}$, and $[\mathrm{AA}]=127 \mu \mathrm{M}$.

Figure 5 shows that the SERS signal of three different Raman-active dyes is further enhanced with laponite-Au nanoparticles as compared with nanostars without laponite. The relative SERS enhancements of the laponite-Au nanoparticles compared with the seed-mediated nanostars for 1-NaT, 2-NaT, and 4-MBA were 30, 3, and 8, respectively. With a calculated enhancement factor (EF) of $\sim 10^{5}$ for $\mathrm{Au}$ nanostars with MBA at 785 $\mathrm{nm}$, we can indirectly estimate the EF for laponite-Au particles at $\sim 10^{6} .^{47}$ In addition, comparison of SERS signal between $633 \mathrm{~nm}$ and $785 \mathrm{~nm}$ excitation was made for crystal violet (CV) and MBA. With MBA, the SERS signal was higher for laponite-Au particles than for nanostars at both excitation wavelengths. In the case of $\mathrm{CV}$, there was greater signal for lap-Au NPs at $785 \mathrm{~nm}$ excitation, but at $633 \mathrm{~nm}$ excitation a high background was observed (Figure S6, supporting information). It has been recently reported that the intercalation of $\mathrm{CV}$ with laponite gives rise to increased fluorescence, ${ }^{48}$ meaning that the anionic faces of the laponite discs are still capable of small molecule adsorption, which may be related to the improved SERS sensitivity for the various dyes studied. If molecules can still adsorb onto bare clay, i.e. clay that is not covered by gold, in the vicinity of the AuNP, then they can experience the enhanced local electric fields generated by the plasmon. This would not only explain the improved SERS, but reveals that these particles may be useful for studying the interactions between aggregated molecular species and plasmon fields. The particles of various morphologies synthesized in the presence of CTAC gave lower SERS enhancements than the 
surfactant-free particles. CTAC-coated particles with shorter gold extrusions (Figure 3B) and longer extrusions (Figure 3C) yielded $\sim 15 \%$ and $\sim 4 \%$ of the enhancement by those in Figure 5, respectively. Surfactant-free particles that were synthesized with overnight stirring yielded SERS enhancements of the same order of magnitude as those synthesized with sonication, and furthermore, better SERS enhancements were observed for particles with a higher lap:Au ratio (Figure S3E,F). Future studies will focus on the formation of molecular aggregates and studying the interaction of their excitons and the gold plasmon, as well as provide a means to study changes in SERS spectra when the Raman dyes are in an aggregated state.

\section{Conclusions and Outlook}

The synthesis of anisotropic gold nanoparticles was directed by the use of the anionic layered silicate clay laponite $\mathrm{RD}$, both with and without the use of surfactant. The role of the clay particle in defining the final shape of the gold-clay nanoparticle was explored through varying experimental parameters such as reagent concentrations, $\mathrm{pH}$, and type of mixing (stirring vs. sonication). Spectroscopic methods and HAADF-STEM electron tomography gave a thorough view of the particles, which revealed several salient parts of the synthesis. Firstly, the use of CTAC in the synthesis results in particles with a larger average diameter. Second, the formation of extrusions of $\mathrm{Au}$ away from the main body of the particles was influenced by both CTAC concentration and rate of reduction (AA concentration). At a fixed AA concentration, increasing CTAC concentration yields a larger number of extrusions. At a given CTAC concentration, the length of the extrusions can increase to more than $100 \mathrm{~nm}$ at low AA, but shorter and more numerous extrusions form with increasing AA. Third, the influence of solution $\mathrm{pH}$ on the edge charge of the laponite discs is reflected by increased particle size and elongation with decreasing $\mathrm{pH}$. Finally, while the majority of syntheses were carried out following mixing of laponite and gold by sonication, the result of metal reduction following overnight stirring of laponite and gold gave a unique particle morphology that likely results from the formation of a cluster of laponite discs intercalated with $\mathrm{Au}$ ions.

Laponite has been previously used for biological applications such as drug delivery and wound dressings, as it has been shown to be nearly harmless against 
mammalian cells. Within the growing field of nanomedicine and theranostics, numerous applications such as hyperthermia and SERS sensing involve the use of IR irradiation due to increased skin penetration depth. This hybrid system of layered silicates and gold allows tailoring of the IR absorption cross-section by two different routes; the control of the length of the extrusions off of the particle, and the control of the pH. Future studies will examine how these highly anisotropic particles with significant extinction in the IR can be utilized for studies of biological systems in vitro. Furthermore, the coupling of plasmonic modes in gold with the ability of laponite to induce dye aggregation will lead to further studies of plasmonic-excitionic systems. Finally, this study has broader implications for possibilities involving other types of layered silicate clays such as montmorillonite and kaolinite. Laponite RD serves as a useful example for study, as it is synthetic and has more uniformity than natural clays. Many of the unique rheological and nanoscopic effects on small-molecule aggregation and strengthening of polymer composites which are enabled by layered silicate clays has yet to be applied in a composite gold-clay system. The use of other surfactants and polymers can lead to different degrees of clay particle delamination, as well as changes in the diffusion of gold chloride to the surface of the clay. While initial studies suggest that different surfactants and polymers lead to different growth kinetics and resulting nanoparticle morphologies correlating with these effects, a comprehensive study is out of the scope of this work. A future direction will explore the use of laponite-gold composites for the formation of solid films or gels for sensing or drug-delivery. Preliminary results show that laponite-Au particles can be formed while retaining similar physical transitions to a gel or semi-gel state that is observed with bare laponite.

Supporting Information. Additional characterization of bare laponite, seed-mediated nanostars, and laponite-gold particles by UV-Visible absorption, TEM, Zeta-potential and hydrodynamic radii, and SERS.

\section{Acknowledgements}

This work has been supported by the European Research Council (ERC Advanced Grant \# 267867, PLASMAQUO). EHH thanks the Spanish Ministry of Economy and Competitiveness for providing a Juan de la Cierva Fellowship (FJCl-2014-22598). N.C. and S.B. acknowledge financial 
support from European Research Council (ERC Starting Grant \#335078-COLOURATOM). We gratefully acknowledge A. B. Serrano-Montes for providing the seed-mediated Au nanostars.

\section{References}

(1) Liz-Marzán, L.M. Tailoring Surface Plasmons through the Morphology and Assembly of Metal Nanoparticles Langmuir 2006, 22, 32-41.

(2) Eustis, S.; el-Sayed, M. a. Why Gold Nanoparticles Are More Precious than Pretty Gold: Noble Metal Surface Plasmon Resonance and Its Enhancement of the Radiative and Nonradiative Properties of Nanocrystals of Different Shapes. Chem. Soc. Rev. 2006, 35, 209-217.

(3) Lee, K.; El-sayed, M. a. Gold and Silver Nanoparticles in Sensing and Imaging: Sensitivity of Plasmon Response to Size, Shape, and Metal Composition Gold and Silver Nanoparticles in Sensing and Imaging : Sensitivity of Plasmon Response to Size, Shape, and Metal Composition. J. Phys. Chem. B 2006, $19220-19225$.

(4) Michaels, A. M.; Brus, L. Ag Nanocrystal Junctions as the Site for SurfaceEnhanced Raman Scattering of Single Rhodamine 6G Molecules. J. Phys. Chem. B 2000, 104, 11965-11971.

(5) Alvarez-Puebla, R. A.; Liz-Marzán, L. M. SERS Detection of Small Inorganic Molecules and Ions. Angew. Chemie - Int. Ed. 2012, 51, 11214-11223.

(6) Zengin, A.; Tamer, U.; Caykara, T. A SERS-Based Sandwich Assay for Ultrasensitive and Selective Detection of Alzheimer's Tau Protein.

Biomacromolecules 2013, 14, 3001-3009.

(7) Li, X.; Chen, G.; Yang, L.; Jin, Z.; Liu, J. Multifunctional Au-Coated TiO2 Nanotube Arrays as Recyclable SERS Substrates for Multifold Organic Pollutants Detection. Adv. Funct. Mater. 2010, 20, 2815-2824.

(8) Costas, C.; López-Puente, V.; Bodelón, G.; González-Bello, C.; Peréz-Juste, J.; 
Pastoriza-Santos, I.; Liz-Marzán, L. M. Using Surface Enhanced Raman Scattering to Analyze the Interactions of Protein Receptors with Bacterial Quorum Sensing Modulators. ACS Nano 2015, 9, 5567-5576.

(9) V. Malgras, Q. Ji, Y. Kamachi, T. Mori, F. K. Shieh, K. C. W. Wu, K. Ariga, Y. Yamauchi Templated Synthesis for Nanoarchitectured Porous Materials Bull.

Chem. Soc. Jpn. 2015, 88, 1171-1200.

(10) Jones, M. R.; Osberg, K. D.; MacFarlane, R. J.; Langille, M. R.; Mirkin, C. A. Templated Techniques for the Synthesis and Assembly of Plasmonic Nanostructures. Chem. Rev. 2011, 111, 3736-3827.

(11) Grzelczak, M.; Perez-Juste, J.; Mulvaney, P.; Liz-Marzán, L. M. Shape Control in Gold Nanoparticle Synthesis. Chem. Soc. Rev. 2008, 37(9), 1783-1791.

(12) Scarabelli, L.; Grzelczak, M.; Liz-Marzán, L. M. Tuning Gold Nanorod Synthesis through Prereduction with Salicylic Acid. Chem. Mater. 2013, 25, 4232-4238.

(13) Scarabelli, L.; Coronado-Puchau, M.; Giner-Casares, J. J.; Langer, J.; LizMarzán, L. M. Monodisperse Gold Nanotriangles : Size Control, Large-Scale Self-Assembly, and Performance in Surface-Enhanced Raman Scattering. ACS Nano 2014, 8(6), 5833-5842.

(14) Liz-Marzán, L. M.; Philipse, A. P. Stable Hydrosols of Metallic and Bimetallic Nanoparticles Immobilized on Imogolite Fibers. J. Phys. Chem. 1995, 99 , $15120-15128$.

(15) Zhang, Z. X.; Van Duijneveldt, J. S. Isotropic-Nematic Phase Transition of Nonaqueous Suspensions of Natural Clay Rods. J. Chem. Phys. 2006, 124, 154910

(16) Paineau, E.; Bihannic, I.; Baravian, C.; Philippe, A.; Davidson, P.; Levitz, P.; Funari, S.; Rochas, C.; Michot, L. J.; Universit, N. Aqueous Suspensions of Natural Swelling Clay Minerals . 1. Structure and Electrostatic Interactions. Langmuir 2011, 27(9), 5562-5573.

(17) Glotzer, S. C.; Solomon, M. J. Anisotropy of Building Blocks and Their Assembly into Complex Structures. Nat. Mater. 2007, 6, 557-562. 
(18) Michot, L. J.; Baravian, C.; Bihannic, I.; Maddi, S.; Moyne, C.; Duval, J. F. L.; Levitz, P.; Davidson, P. Sol-gel and Isotropic/Nematic Transitions in Aqueous Suspensions of Natural Nontronite Clay. Influence of Particle Anisotropy. 2. Gel Structure and Mechanical Properties. Langmuir 2008, 25, 127-139.

(19) Shalkevich, A.; Stradner, A.; Bhat, S. K.; Mulle, F.; Schurtenberger, P. Cluster, Glass, and Gel Formation and Viscoelastic Phase Separation in Aqueous Clay Suspensions. Langmuir 2007, 23, 3570-3580.

(20) Atmuri, A. K.; Bhatia, S. R. Polymer-Mediated Clustering of Charged Anisotropic Colloids. Langmuir 2013, 29, 3179-3187.

(21) Gradzielski, M.; Hoffmann, H.; Oetter, G. Ringing Gels: Their Structure and Macroscopic Properties. Colloid Polym. Sci. 1990, 268(2), 167-178.

(22) Ramsay, J. D. F.; Lindner, P. Small-Angle Neutron Scattering Investigations of the Structure of Thixotropic Dispersions of Smectite Clay Colloids. J. Chem. Soc. Faraday Trans. 1993, 89, 4207-4214.

(23) Luckham, P. F.; Rossi, S. Colloidal and Rheological Properties of Bentonite Suspensions. Adv. Colloid Interface Sci. 1999, 82, 43-92.

(24) Ganley, W. J.; Van Duijneveldt, J. S. Controlling Clusters of Colloidal Platelets: Effects of Edge and Face Surface Chemistries on the Behavior of Montmorillonite Suspensions. Langmuir 2015, 31, 4377-4385.

(25) Martínez Martínez, V.; López Arbeloa, F.; Bañuelos Prieto, J.; Arbeloa López, T.; López Arbeloa, I. Characterization of Supported Solid Thin Films of Laponite Clay. Intercalation of Rhodamine 6G Laser Dye. Langmuir 2004, 20(14), 57095717.

(26) Thomas, J. K. Photophysical and Photochemical Processes on Clay Surfaces. Acc. Chem. Res. 1988, 21(9), 275-280.

(27) Shichi, T.; Takagi, K. Clay Minerals as Photochemical Reaction Fields. J. Photochem. Photobiol. C Photochem. Rev. 2000, 1(2), 113-130.

(28) Hill, E. H.; Zhang, Y.; Whitten, D. G. Aggregation of Cationic $p$-Phenylene Ethynylenes on Laponite Clay in Aqueous Dispersions and Solid Films. $J$. Colloid Interface Sci. 2015, 449, 347-356. 
(29) Chen, G.; Li, D.; Li, J.; Cao, X.; Wang, J.; Shi, X.; Guo, R. Targeted

Doxorubicin Delivery to Hepatocarcinoma Cells by Lactobionic Acid-Modified Laponite Nanodisks. New J. Chem. 2015, 39, 2847-2855.

(30) Joshi, G. V; Kevadiya, B. D.; Patel, H.; Bajaj, H. C.; Jasra, R. V.

Montmorillonite as a Drug Delivery System: Intercalation and in Vitro Release of timolol maleate. Int. J. Pharm. 2009, 374, 53-57.

(31) Ghadiri, M.; Hau, H.; Chrzanowski, W.; Agus, H.; Rohanizadeh, R. Laponite Clay as a Carrier for in Situ Delivery of Tetracycline. $R S C A d v . \mathbf{2 0 1 3}, 3(43)$, 20193.

(32) Donauerová, A.; Bujdák, J.; Smolinská, M.; Bujdáková, H. Photophysical and Antibacterial Properties of Complex Systems Based on Smectite, a Cationic Surfactant and Methylene Blue. J. Photochem. Photobiol. B. 2015, 151, 135-141.

(33) Ghadiri, M.; Chrzanowski, W.; Rohanizadeh, R. Biomedical Applications of Cationic Clay Minerals. RSC Adv. 2015, 5, 29467-29481.

(34) Anonymous, Laponite Technical Bulletin, L104/90/A, 1, (1990)

(35) Avery, R. G.; Ramsay, J. D. F. Colloidal Properties of Synthetic Hectorite Clay Dispersions. II. Light and Small Angle Neutron Scattering. J. Colloid Interface Sci. 1986, 109, 448-454.

(36) Thompson, D. W.; Butterworth, J. T. The Nature of Laponite and Its Aqueous Dispersions J. Colloid Interface Sci. 1992, 15, 236-243.

(37) Yuan, H.; Khoury, C. G.; Hwang, H.; Wilson, C. M.; Grant, G. A.; Vo-Dinh, T. Gold Nanostars: Surfactant-Free Synthesis, 3D Modelling, and Two-Photon Photoluminescence Imaging. Nanotechnology 2012, 23, 75102.

(38) Ojea-Jiménez, I.; Campanera, J. Molecular Modeling of the Reduction Mechanism in the Citrate-Mediated Synthesis of Gold Nanoparticles. J. Phys. Chem. C 2012, 116, 23682-23691.

(39) Nakamura, T.; Thomas, J. K. The Interaction of Alkylammonium Salts with Synthetic Clays. A Fluorescence and Laser Excitation Study. J. Phys. Chem. 1986, 90, 641-644. 
(40) B. Wang, M. Zhou, Z. Rozynek, J. O. Fossum Electrorheological Properties of Organically Modified Nanolayered Laponite: Influence of Intercalation, Adsorption, and Wettability J. Mater. Chem. 2009, 19, 1816.

(41) Angelomé, P. C.; Mezerji, H. H.; Goris, B.; Pastoriza-Santos, I.; Pérez-Juste, J.; Bals, S.; Liz-Marzán, L. M. Seedless Synthesis of Single Crystalline Au Nanoparticles with Unusual Shapes and Tunable LSPR in the near-IR. Chem. Mater. 2012, 24, 1393-1399.

(42) Ebrahimi, D.; Whittle, A. J.; Pellenq, R. J. Mesoscale Properties of Clay Aggregates from Potential of Mean Force Representation of Interactions between Nanoplatelets. J. Chem. Phys. 2014, 140, 154309.

(43) Pek-Ing, A.; Yee-Kwong, L. Surface Chemistry and Rheology of Laponite Dispersions - Zeta Potential, Yield Stress, Ageing, Fractal Dimension and Pyrophosphate. Appl. Clay Sci. 2015, 107, 36-45.

(44) Pal, T.; De, S.; Jana, N. R.; Pradhan, N.; Mandal, R.; Pal, A.; Beezer, A. E.; Mitchell, J. C. Organized Media as Redox Catalysts. Langmuir 1998, 14, 47244730 .

(45) Alvarez-Puebla, R. A.; Dos Santos, D. S.; Aroca, R. F. Surface-Enhanced Raman Scattering for Ultrasensitive Chemical Analysis of 1 and 2-Naphthalenethiols. Analyst 2004, 129, 1251-1256.

(46) Michota, A.; Bukowska, J. Surface-Enhanced Raman Scattering (SERS) of 4Mercaptobenzoic Acid on Silver and Gold Substrates. J. Raman Spectrosc. 2003, $34,21-25$.

(47) Serrano-Montes, A. B.; De Aberasturi, D. J.; Langer, J.; Giner-Casares, J. J.; Scarabelli, L.; Herrero, A.; Liz-Marzán, L. M. A General Method for Solvent Exchange of Plasmonic Nanoparticles and Self-Assembly into SERS-Active Monolayers. Langmuir 2015, 31, 9205-9213.

(48) Ley, C.; Brendlé, J.; Walter, A.; Jacques, P.; Ibrahim, A.; Allonas, X. On the Interaction of Triarylmethane Dye Crystal Violet with Laponite Clay: Using Mineral Nanoparticles to Control the Dye Photophysics. Phys. Chem. Chem. Phys. 2015, 17, 16677-16681. 
TOC graphic

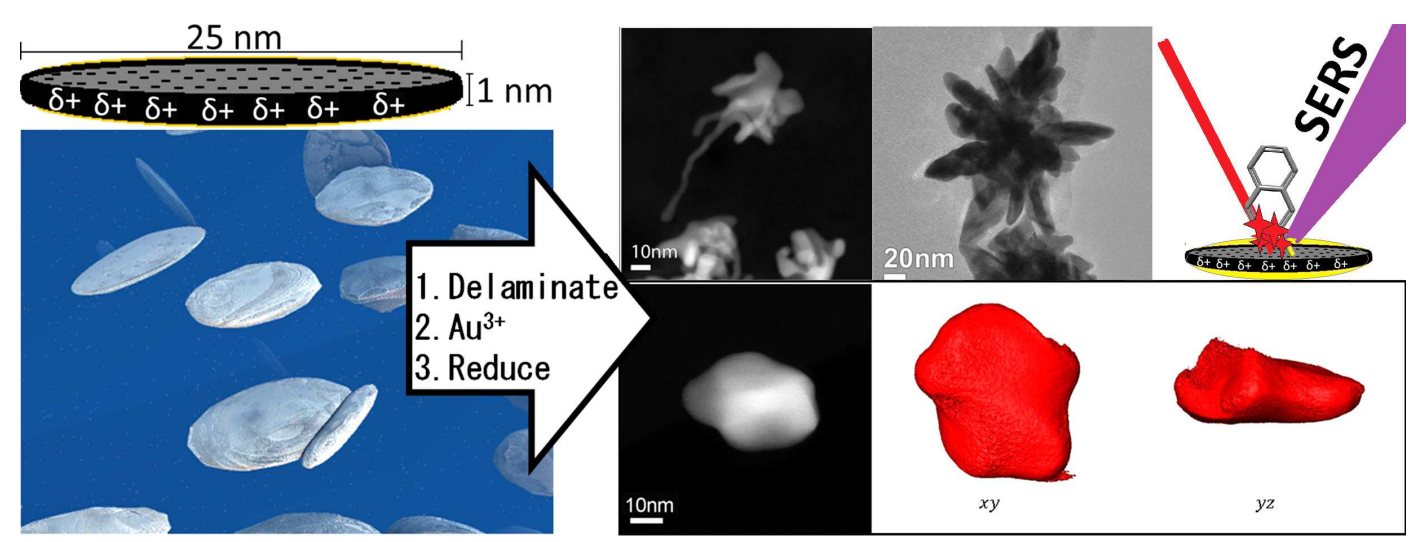

29

30

31 